\title{
BOUNDARY VALUE PROBLEMS SINGULAR IN THE SOLUTION VARIABLE WITH NONLINEAR BOUNDARY DATA
}

\author{
by DONAL O'REGAN
}

(Received 3rd November 1994)

Existence results are established for the equation $y^{\prime \prime}+f(t, y)=0,0<t<1$. Here $f$ may be singular in $y$ and $f$ is allowed to change sign. Our boundary data include $y(0)=y^{\prime}(1)+k y(1)=0, k>-1$ and $y(0)=y^{\prime}(1)+c y^{4}(1)=0$, $c>0$.

1991 Mathematics subject classification: 34B15.

\section{Introduction}

This paper discusses problems of the form

$$
\left\{\begin{array}{l}
y^{\prime \prime}+f(t, y)=0,0<t<1 \\
y(0)=0 \\
y^{\prime}(1)+\mu \psi(y(1))=0, \mu \geqq 0 \text { a constant }
\end{array}\right.
$$

where $f$ is not a Carathéodory function due to the singular behavior of its $y$ variable. Here $\psi$ may be nonlinear and includes for example the Sturm Liouville boundary condition $y^{\prime}(1)+k y(1)=0, k>-1$ and Stefan's condition $y^{\prime}(1)+c y^{4}(1)=0, c>0$. Also our nonlinearity $f$ is allowed to change sign.

Our study is motivated by the problem

$$
\left\{\begin{array}{l}
y^{\prime \prime}+\left(\frac{t^{2}}{32 y^{2}}-\frac{\kappa^{2}}{8}\right)=0,0<t<1 \\
y(0)=0 \\
2 y^{\prime}(1)-(1+v) y(1)=0, \kappa>0 \text { and } 0<v<1
\end{array}\right.
$$

which arises in nonlinear mechanics; see $[1,9]$ and their references. The problem models the stress in the spherical membrane of a spherical cap.

The literature $[2-4,7-8,10-12]$ on singular problems of the above type is almost totally devoted to the Dirichlet problem 


$$
\left\{\begin{array}{l}
y^{\prime \prime}+f(t, y)=0,0<t<1 \\
y(0)=0=y(1)
\end{array}\right.
$$

usually when $f(t, y) \geqq 0$ for $t \in(0,1)$ and $y>0$. Very little seems to be known concerning the class of problems (1.1), which includes (1.2). In this paper we obtain a general existence theory for problems of the form (1.1).

The analysis used throughout rely on fixed point methods. We state, for convenience, the two fixed point theorems we will use.

Theorem 1.1. (Schauder [11]). Let $K$ be a convex subset of a normed linear space $E$. Then every compact map $F: K \rightarrow K$ has at least one fixed point.

Theorem 1.2. (Nonlinear Alternative [5, 11]). Assume $U$ is a relatively open subset of a convex set $K$ in a normed linear space $E$. Let $N: \bar{U} \rightarrow K$ be a compact map with $p \in U$. Then either

(i) $N$ has a fixed point in $\bar{U}$; or

(ii) there is $a u \in \partial U$ and $a \lambda \in(0,1)$ such that $u=\lambda N u+(1-\lambda) p$.

Remark. By a map being compact we mean it is continuous with relatively compact range. For later purposes, a map is completely continuous if it is continuous and the image of every bounded set in the domain is contained in a compact set in the range.

\section{Existence}

Several existence results are presented for the singular problem

$$
\left\{\begin{array}{l}
y^{\prime \prime}+f(t, y)=0,0<t<1 \\
y(0)=0 \\
y^{\prime}(1)+\mu \psi(y(1))=0, \mu \geqq 0 \text { a constant. }
\end{array}\right.
$$

Our first two results were motivated by the boundary value problem (1.2); in particular by the boundary condition $2 y^{\prime}(1)-(1+v) y(1)=0$. By a solution to (2.1) we mean a function $y \in C[0,1] \cap C^{1}(0,1] \cap C^{2}(0,1)$ which satisfies the differential equation on $(0,1)$ and the stated boundary data.

Theorem 2.1. Suppose the following conditions are satisfied:

$$
\begin{gathered}
f:(0,1) \times(0, \infty) \rightarrow \mathbf{R} \text { is continuous } \\
\psi: \mathbf{R} \rightarrow \mathbf{R} \text { is continuous with } \psi(x) \leqq 0 \text { for } x \geqq 0
\end{gathered}
$$




$$
\left\{\begin{array}{l}
|f(t, y)| \leqq q_{1}(t) g(y)+q_{2}(t) h(y) \text { on }(0,1) \times(0, \infty) \text { with } g>0 \\
\text { continuous and nonincreasing on }(0, \infty), h \geqq 0 \text { continuous } \\
\text { on }[0, \infty) \text { and } \frac{h}{g} \text { nondecreasing on }(0, \infty) ; \text { here } q_{i} \in C(0,1), \\
i=1,2 \text { with } q_{i}>0 \text { on }(0,1) \text { and } \int_{0}^{1} q_{i}(x) d x<\infty
\end{array}\right.
$$

$\left\{\begin{array}{l}\text { let } n \in\{3,4, \ldots\} \text { and associated with each } n \text { we have a constant } \\ \rho_{n} \text { such that }\left\{\rho_{n}\right\} \text { is a nonincreasing sequence with } \lim _{n \rightarrow \infty} \rho_{n}=0 \\ \text { and such that for } \frac{1}{n} \leqq t \leqq 1-\frac{1}{n} \text { we have } f\left(t, \rho_{n}\right) \geqq 0\end{array}\right.$

$$
\begin{aligned}
& \left\{\begin{array}{l}
\text { there exists a function } \alpha \in C[0,1] \cap C^{1}(0,1] \cap C^{2}(0,1) \text { with } \\
\alpha(0)=\alpha^{\prime}(1)+\mu \psi(\alpha(1))=0, \alpha>0 \text { on }(0,1) \text { such that } \\
f(t, y)+\alpha^{\prime \prime}(t)>0 \text { for }(t, y) \in(0,1) \times\{y \in(0, \infty): y<\alpha(t)\}
\end{array}\right. \\
& \qquad \int_{1 / 2}^{1} q_{i}(x) g(\alpha(x)) d x<\infty, \quad u=1,2 \\
& \left\{\begin{array}{l}
\text { for any } R>0, \frac{1}{g} \text { is differentiable on }(0, R] \text { with } g^{\prime}<0 \text { a.e. } \\
\text { and } \frac{g^{\prime}}{g^{2}} \in L^{1}[0, R] ; \text { in addition } \int_{0}^{\infty} \frac{\left|g^{\prime}(t)\right|^{1 / 2}}{g(t)} d t=\infty
\end{array}\right.
\end{aligned}
$$

and

$$
\left\{\begin{array}{l}
\text { there exists a constant } M>0 \text { such that for } z>0, \\
\int_{0}^{z} \frac{d u}{g(u)} \leqq \frac{\left[\rho_{3}+\mu\left|\psi^{*}(z)\right|\right]}{g(z)}+\int_{0}^{1} x q_{1}(x) d x+\frac{h(z)}{g(z)} \int_{0}^{1} x q_{2}(x) d x+\int_{0}^{\rho_{3}} \frac{d u}{g(u)} \\
\text { implies } z \leqq M ;
\end{array}\right.
$$

here

$$
\psi^{*}(z)=\left\{\begin{array}{l}
\psi(z), z \geqq \alpha(1) \\
\psi(\alpha(1)), z<\alpha(1)
\end{array}\right.
$$

Then (2.1) has a solution in $C[0,1] \cap C^{1}(0,1] \cap C^{2}(0,1)$.

Remark. Note $\psi^{*}(z) \leqq 0$ for $z \in \mathbf{R}$.

Proof. Fix $n \in\{3,4, \ldots\}$. We begin by showing that 


$$
\left\{\begin{array}{l}
y^{\prime \prime}+f(t, y)=0,0<t<1 \\
y(0)=\rho_{n} \\
y^{\prime}(1)+\mu \psi(y(1))=\rho_{n}
\end{array}\right.
$$

has a solution in $C^{1}[0,1] \cap C^{2}(0,1)$. To show $(2.10)^{n}$ has a solution we consider the family of problems

$$
\left\{\begin{array}{l}
y^{\prime \prime}+\lambda f^{*}(t, y)=0,0<t<1,0<\lambda<1 \\
y(0)=\rho_{n} \\
y^{\prime}(1)+\lambda \mu \psi^{*}(y(1))=\rho_{n}
\end{array}\right.
$$

where

$$
f^{*}(t, y)=\left\{\begin{array}{l}
f(t, y), y \geqq \rho_{n} \\
f\left(t, \rho_{n}\right)+\rho_{n}-y, y<\rho_{n} \text { and } \frac{1}{n} \leqq t \leqq 1-\frac{1}{n} \\
f\left(\frac{1}{n}, \rho_{n}\right)+\rho_{n}-y, y<\rho_{n} \text { and } 0 \leqq t \leqq \frac{1}{n} \\
f\left(1-\frac{1}{n}, \rho_{n}\right)+\rho_{n}-y, y<\rho_{n} \text { and } 1-\frac{1}{n} \leqq t \leqq 1
\end{array}\right.
$$

We first show that

$$
y(t) \geqq \rho_{n}, \quad t \in[0,1]
$$

for any solution $y \in C^{1}[0,1] \cap C^{2}(0,1)$ to $(2.11)_{\lambda}^{n}$. To see this suppose $y-\rho_{n}$ has a negative minimum at $t_{0} \in(0,1]$. If $t_{0} \in(0,1)$ then $y^{\prime}\left(t_{0}\right)=0$ and $y^{\prime \prime}\left(t_{0}\right) \geqq 0$. However

$$
y^{\prime \prime}\left(t_{0}\right)=-\lambda f^{*}\left(t_{0}, y\left(t_{0}\right)\right)=\left\{\begin{array}{l}
-\lambda\left[f\left(t_{0}, \rho_{n}\right)+\rho_{n}-y\left(t_{0}\right)\right] \text { if } \frac{1}{n} \leqq t_{0} \leqq 1-\frac{1}{n} \\
-\lambda\left[f\left(\frac{1}{n}, \rho_{n}\right)+\rho_{n}-y\left(t_{0}\right)\right] \text { if } 0 \leqq t_{0} \leqq \frac{1}{n} \\
-\lambda\left[f\left(1-\frac{1}{n}, \rho_{n}\right)+\rho_{n}-y\left(t_{0}\right)\right] \text { if } 1-\frac{1}{n} \leqq t_{0} \leqq 1
\end{array}\right.
$$

i.e. $y^{\prime \prime}\left(t_{0}\right)<0$, a contradiction. It remains to consider the case $t_{0}=1$. Then $y^{\prime}(1) \leqq 0$. However

$$
y^{\prime}(1)=\rho_{n}-\lambda \mu \psi^{*}(y(1))>0,
$$

a contradiction. Thus (2.12) holds.

Suppose the absolute maximum of $y$ occurs at say $t_{n}$. Note we take $t_{n} \in(0,1]$. There are two cases to consider, namely $t_{n} \in(0,1)$ and $t_{n}=1$. Notice for $x \in(0,1)$ we have 


$$
\frac{-y^{\prime \prime}(x)}{g(y(x))} \leqq q_{1}(x)+q_{2}(x) \frac{h(y(x))}{g(y(x))}
$$

Case (i) $t_{n} \in(0,1)$.

Then $y^{\prime}\left(t_{n}\right)=0$. Integrate (2.13) from $t\left(t<t_{n}\right)$ to $t_{n}$ to obtain

$$
\frac{y^{\prime}(t)}{g(y(t))}+\int_{t}^{t_{n}}\left\{\frac{-g^{\prime}(y(x))}{g^{2}(y(x))}\right\}\left[y^{\prime}(x)\right]^{2} d x \leqq \int_{t}^{t_{n}} q_{1}(x) d x+\frac{h\left(y\left(t_{n}\right)\right)}{g\left(y\left(t_{n}\right)\right)} \int_{t}^{t_{n}} q_{2}(x) d x
$$

and so

$$
\frac{y^{\prime}(t)}{g(y(t))} \leqq \int_{t}^{t_{n}} q_{1}(x) d x+\frac{h\left(y\left(t_{n}\right)\right)}{g\left(y\left(t_{n}\right)\right)} \int_{t}^{t_{n}} q_{2}(x) d x
$$

Integrate from 0 to $t_{n}$ to obtain

$$
\int_{0}^{y\left(t_{n}\right)} \frac{d u}{g(u)} \leqq \int_{0}^{1} x q_{1}(x) d x+\frac{h\left(y\left(t_{n}\right)\right)}{g\left(y\left(t_{n}\right)\right)} \int_{0}^{1} x q_{2}(x) d x+\int_{0}^{\rho_{3}} \frac{d u}{g(u)}
$$

Consequently (2.9) implies

$$
y\left(t_{n}\right) \leqq M
$$

Case (ii) $t_{n}=1$.

Now since $y^{\prime}(1)=\rho_{n}-\lambda \mu \psi^{*}(y(1))$ we have

$$
\left|y^{\prime}(1)\right| \leqq \rho_{3}+\mu\left|\psi^{*}(y(1))\right| .
$$

Integrate (2.13) from $t$ to 1 to obtain

$$
\frac{y^{\prime}(t)}{g(y(t))}-\frac{y^{\prime}(1)}{g(y(1))} \leqq \int_{t}^{1} q_{1}(x) d x+\frac{h(y(1))}{g(y(1))} \int_{t}^{1} q_{2}(x) d x,
$$

and so

$$
\frac{y^{\prime}(t)}{g(y(t))} \leqq \frac{\left.\left[\rho_{3}+\mu \mid \psi^{*}(y(1))\right]\right]}{g(y(1))}+\int_{t}^{1} q_{1}(x) d x+\frac{h(y(1))}{g(y(1))} \int_{t}^{1} q_{2}(x) d x
$$

Integrate from 0 to 1 to obtain

$$
\int_{0}^{y\left(t_{n}\right)} \frac{d u}{g(u)} \leqq \frac{\left.\left[\rho_{3}+\mu \mid \psi^{*}(y(1))\right]\right]}{g(y(1))}+\int_{0}^{1} x q_{1}(x) d x+\frac{h(y(1))}{g(y(1))} \int_{0}^{1} x q_{2}(x) d x+\int_{0}^{\rho_{3}} \frac{d u}{g(u)} .
$$

Consequently (2.9) implies

$$
y\left(t_{n}\right)=y(1) \leqq M
$$


Thus in both cases

$$
\rho_{n} \leqq y(t) \leqq M \text { for } t \in[0,1]
$$

Also the mean value theorem implies that there exists $\tau \in(0,1)$ with $\left|y^{\prime}(\tau)\right|=|y(1)-y(0)|$ $\leqq 2 M$. For $t \in[0,1]$ we have

$$
\begin{aligned}
\left|y^{\prime}(t)\right| & \leqq\left|y^{\prime}(\tau)\right|+\left|\int_{t}^{t}\right| f^{*}(x, y(x))|d x| \\
& \leqq 2 M+g\left(\rho_{n}\right) \int_{0}^{1}\left[q_{1}(x)+q_{2}(x) \frac{h(M)}{g(M)}\right] d x \equiv M_{1} .
\end{aligned}
$$

Define the mappings

$$
L, F: C_{\rho_{n}}^{1}[0,1] \rightarrow C_{0}[0,1] \times \mathbf{R}
$$

by

$$
L y(t)=\left(y^{\prime}(t)-y^{\prime}(0), \rho_{n}-y^{\prime}(1)\right) \quad \text { and } \quad F y(t)=\left(-\int_{0}^{t} f^{*}(x, y(x)) d x, \mu \psi^{*}(y(1))\right)
$$

Here $C_{0}[0,1]=\{u \in C[0,1]: u(0)=0\}$ and $C_{\rho_{n}}^{1}[0,1]=\left\{u \in C^{1}[0,1]: u(0)=\rho_{n}\right\}$. Now $F$ is completely continuous by the Arzela-Ascoli theorem. Also if $L y=(u(t), \gamma)$ then

$$
y(t)=\rho_{n}+\left(\rho_{n}-\gamma-u(1)\right) t+\int_{0}^{t} u(x) d x
$$

hence $L^{-1}$ exists and is continuous.

Solving (2.11) ${ }_{\lambda}^{n}$ is equivalent to finding a fixed point of $y=\lambda L^{-1} F y=\lambda N y$ where $N=L^{-1} F: C_{\rho_{n}}^{1}[0,1] \rightarrow C_{\rho_{n}}^{1}[0,1]$ is completely continuous. Let

$$
U=\left\{u \in C_{\rho_{n}}^{1}[0,1]:|u|_{1}<\max \left\{M, M_{1}\right\}+1\right\}, K=C_{\rho_{n}}^{1}[0,1] \text { and } E=C^{1}[0,1]
$$

here $|u|_{1}=\max \left\{|u|_{0},\left|u^{\prime}\right|_{0}\right\}$ and $|u|_{0}=\sup _{[0,1]}|u(t)|$. Now Theorem 1.2 implies that $(2.11)_{1}^{n}$ has a solution $y_{n} \in C^{1}[0,1] \cap C^{2}(0,1)$. Also $\rho_{n} \leqq y_{n}(t) \leqq M$ for $t \in[0,1]$. Next we obtain a sharper lower bound on $y_{n}$, namely we will show

$$
\alpha(t) \leqq y_{n}(t) \leqq M \quad \text { for } t \in[0,1]
$$

If this is not true then $y_{n}-\alpha$ would have a negative minimum at say $t_{0} \in(0,1]$. If $t_{0} \in(0,1)$ then $y_{n}^{\prime \prime}\left(t_{0}\right)-\alpha^{\prime \prime}\left(t_{0}\right) \geqq 0$. However since $0<y_{n}\left(t_{0}\right)<\alpha\left(t_{0}\right)$ and $y_{n}\left(t_{0}\right) \geqq \rho_{n}$ we have

$$
y_{n}^{\prime \prime}\left(t_{0}\right)-\alpha^{\prime \prime}\left(t_{0}\right)=-\left[f\left(t_{0}, y_{n}\left(t_{0}\right)\right)+\alpha^{\prime \prime}\left(t_{0}\right)\right]<0,
$$


a contradiction. It remains to consider the case $t_{0}=1$. Then $y_{n}^{\prime}(1) \leqq \alpha^{\prime}(1)$ and $0<y_{n}(1)<$ $\alpha(1)$. However

$$
\begin{aligned}
y_{n}^{\prime}(1)-\alpha(1) & =\rho_{n}-\mu \psi^{*}(y(1))+\mu \psi(\alpha(1)) \\
& =\rho_{n}-\mu \psi(\alpha(1))+\mu \psi(\alpha(1))=\rho_{n}>0,
\end{aligned}
$$

a contradiction. Hence (2.17) is true. In particular $y_{n}(1) \geqq \alpha(1)$ and consequently $y_{n} \in C^{1}[0,1] \cap C^{2}(0,1)$ is a solution of $(2.10)^{n}$.

We shall now obtain a solution to (2.1) by means of the Arzela-Ascoli theorem, as a limit of solutions of $(2.10)^{n}$. To this end, we will show

$$
\left\{y_{n}\right\}_{n=3}^{\infty} \text { is a bounded, equicontinuous family on }[0,1] .
$$

Of course $\left\{y_{n}\right\}$ is uniformly bounded by (2.17). To show equicontinuity, some more estimates are needed.

The differential equation yields

$$
-y_{n}^{\prime \prime}(x) \leqq g\left(y_{n}(x)\right)\left\{q_{1}(x)+q_{2}(x) \frac{h(M)}{g(M)}\right\} \text { for } \quad x \in(0,1) .
$$

Also $y_{n}^{\prime}(1)=\rho_{n}-\mu \psi\left(y_{n}(1)\right)$ together with (2.17) yields

$$
\left|y_{n}^{\prime}(1)\right| \leqq \rho_{3}+\mu \max _{z \in[0, M]}|\psi(z)| \equiv K_{0} .
$$

Divide (2.19) by $g\left(y_{n}(x)\right)$ and integrate from 0 to 1 to obtain

$$
\frac{-y_{n}^{\prime}(1)}{g\left(y_{n}(1)\right)}+\frac{y_{n}^{\prime}(0)}{g\left(\rho_{n}\right)}+\int_{0}^{1}\left\{\frac{-g^{\prime}\left(y_{n}(x)\right)}{g^{2}\left(y_{n}(x)\right)}\right\}\left[y_{n}^{\prime}(x)\right]^{2} d x \leqq \int_{0}^{1}\left[q_{1}(x)+q_{2}(x) \frac{h(M)}{g(M)}\right] d x .
$$

Then since $y_{n}^{\prime}(0) \geqq 0\left(\right.$ note $y_{n}(0)=\rho_{n}$ and $y_{n} \geqq \rho_{n}$ on $\left.[0,1]\right)$ we have

$$
\int_{0}^{1}\left\{\frac{-g^{\prime}\left(y_{n}(x)\right)}{g^{2}\left(y_{n}(x)\right)}\right\}\left[y_{n}^{\prime}(x)\right]^{2} d x \leqq \frac{K_{0}}{g(M)}+\int_{0}^{1}\left[q_{1}(x)+q_{2}(x) \frac{h(M)}{g(M)}\right] d x \equiv K_{1} .
$$

Now consider

$$
I(z)=\int_{0}^{z} \frac{\left[-g^{\prime}(u)\right]^{1 / 2}}{g(u)} d u .
$$

Notice $I$ is an increasing map from $[0, \infty)$ onto $[0, \infty)$ with $I$ continuous on $[0, \Omega]$ for any $\Omega>0$. For $t, s \in[0,1]$ we have from Hölder's inequality that 


$$
\begin{aligned}
\left|I\left(y_{n}(t)\right)-I\left(y_{n}(s)\right)\right| & =\left|\int_{y_{n}(s)}^{y_{n}(t)} \frac{\left[-g^{\prime}(u)\right]^{1 / 2}}{g(u)} d u\right|=\left|\int_{s}^{t} \frac{\left[-g^{\prime}\left(y_{n}(x)\right)\right]^{1 / 2}}{g\left(y_{n}(x)\right)} y_{n}^{\prime}(x) d x\right| \\
& \leqq|t-s|^{1 / 2}\left(\int_{0}^{1}\left\{\frac{-g^{\prime}\left(y_{n}(x)\right)}{g^{2}\left(y_{n}(x)\right)}\right\}\left[y_{n}^{\prime}(x)\right]^{2} d x\right)^{1 / 2} \leqq K_{1}^{1 / 2}|t-s|^{1 / 2}
\end{aligned}
$$

It follows from this inequality, the uniform continuity of $I^{-1}$ on $[0, I(M)]$ and

$$
\left|y_{n}(t)-y_{n}(s)\right|=\left|I^{-1}\left(I\left(y_{n}(t)\right)\right)-I^{-1}\left(I\left(y_{n}(s)\right)\right)\right|
$$

that $\left\{y_{n}\right\}$ is equicontinuous on $[0,1]$. Thus $(2.18)$ is established.

The Arzela-Ascoli theorem guarantees the existence of a subsequence $N$ of integers and a function $y \in C[0,1]$ with $y_{n}$ converging uniformly on $[0,1]$ to $y$ as $n \rightarrow \infty$ through $N$. Also $y(0)=0$ and $\alpha(t) \leqq y(t) \leqq M$ for $t \in[0,1]$. Now $y_{n}, n \in N$, satisfies the integral equation

$$
y_{n}(t)=y_{n}(1)+\left(\mu \psi\left(y_{n}(1)\right)-\rho_{n}\right)(1-t)-\int_{t}^{1}(x-t) f\left(x, y_{n}(x)\right) d x \quad \text { for } \quad t \in[0,1]
$$

We would like to let $n \rightarrow \infty$ through $N$ in (2.22). First notice

$$
\int_{1 / 2}^{1}\left|f\left(x, y_{n}(x)\right)\right| d x \leqq \int_{1 / 2}^{1} g(\alpha(x))\left\{q_{1}(x)+q_{2}(x) \frac{h(M)}{g(M)}\right\} d x<\infty
$$

Fix $t \in(0,1]$. Let $n \rightarrow \infty$ through $N$ in (2.22), and so the Lebesgue dominated convergence theorem implies

$$
y(t)=y(1)+\mu \psi(y(1))(1-t)-\int_{t}^{1}(x-t) f(x, y(x)) d x .
$$

Also for $t \in(0,1]$ we have

$$
y^{\prime}(t)=-\mu \psi(y(1))+\int_{t}^{1} f(x, y(x)) d x
$$

so $y \in C^{1}(0,1]$. In addition $-y^{\prime \prime}(t)=f(t, y(t))$ for $t \in(0,1)$ and $y \in C^{2}(0,1)$. Finally (2.23) implies $y^{\prime}(1)+\mu \psi(y(1))=0$.

The next theorem is a "general upper and lower solution theorem" for singular problems with nonlinear boundary data.

Theorem 2.2. Suppose (2.2)-(2.8) hold. In addition assume 


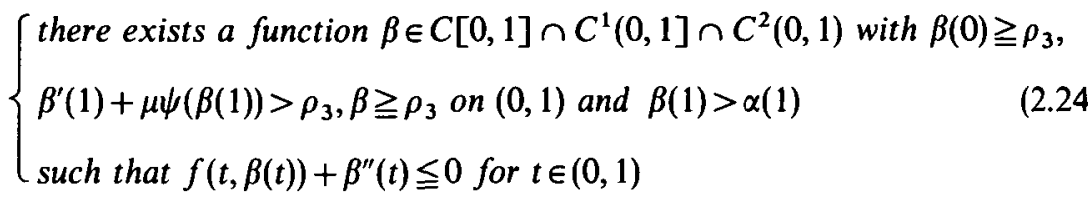

is satisfied. Then (2.1) has a solution in $C[0,1] \cap C^{1}(0,1] \cap C^{2}(0,1)$.

Proof. Fix $n \in\{3,4, \ldots\}$. We first show

$$
\left\{\begin{array}{l}
y^{\prime \prime}+f(t, y)=0,0<t<1 \\
y(0)=\rho_{n} \\
y^{\prime}(1)+\mu \psi(y(1))=\rho_{n}
\end{array}\right.
$$

has a solution. The idea is to look at

$$
\left\{\begin{array}{l}
y^{\prime \prime}+f^{* *}(t, y)=0,0<t<1 \\
y(0)=\rho_{n} \\
y^{\prime}(1)+\mu \psi^{* *}(y(1))=\rho_{n}
\end{array}\right.
$$

where

$$
\begin{gathered}
f^{* *}(t, y)=\left\{\begin{array}{l}
f(t, \beta(t))+r(\beta(t)-y), y \geqq \beta(t) \\
f(t, y), \rho_{n} \leqq y \leqq \beta(t) \\
f\left(t, \rho_{n}\right)+r\left(\rho_{n}-y\right), y<\rho_{n} \text { and } \frac{1}{n} \leqq t \leqq 1-\frac{1}{n} \\
f\left(\frac{1}{n}, \rho_{n}\right)+r\left(\rho_{n}-y\right), y<\rho_{n} \text { and } 0 \leqq t \leqq \frac{1}{n} \\
f\left(1-\frac{1}{n}, \rho_{n}\right)+r\left(\rho_{n}-y\right), y<\rho_{n} \text { and } 1-\frac{1}{n} \leqq t \leqq 1
\end{array}\right. \\
\psi^{* *}(z)=\left\{\begin{array}{l}
\psi(\beta(1)), z>\beta(1) \\
\psi(z), \alpha(1) \leqq z \leqq \beta(1) \\
\psi(\alpha(1)), z<\alpha(1) .
\end{array}\right.
\end{gathered}
$$

and $r: \mathbf{R} \rightarrow[-1,1]$ is the radial retraction defined by

$$
r(u)= \begin{cases}u & \text { if }|u| \leqq 1 \\ \frac{u}{|u|} & \text { otherwise }\end{cases}
$$


Remark. Notice $\psi^{* *}(z) \leqq 0$ for $z \in \mathbf{R}$.

Let $C_{0}[0,1], C_{\rho_{n}}^{1}[0,1]$ be as in Theorem 2.1 and define mappings

$$
L, F: C_{\rho_{n}}^{1}[0,1] \rightarrow C_{0}[0,1] \times \mathbf{R}
$$

by

$$
L y(t)=\left(y^{\prime}(t)-y^{\prime}(0), \rho_{n}-y^{\prime}(1)\right) \quad \text { and } \quad F y(t)=\left(-\int_{0}^{1} f^{* *}(x, y(x)) d x, \mu \psi^{* *}(y(1))\right)
$$

Now $L^{-1}$ exists and is continuous as in Theorem 2.1. Notice also that $F$ is compact. Hence solving (2.26) $)^{n}$ is equivalent to finding a fixed point of $y=L^{-1} F y=N y$ where $N=L^{-1} F: C_{\rho_{n}}^{1}[0,1] \rightarrow C_{\rho_{n}}^{1}[0,1]$ is compact. Theorem 1.1 implies $(2.26)^{n}$ has a solution $y_{n} \in C^{1}[0,1] \cap C^{2}(0,1)$. Essentially the same reasoning as in Theorem 2.1 yields

$$
y_{n}(t) \geqq \rho_{n} \quad \text { for } \quad t \in[0,1]
$$

Next we claim

$$
y_{n}(t) \leqq \beta(t) \text { for } t \in[0,1]
$$

If (2.28) is not true then $y_{n}-\beta$ would have a positive maximum at say $t_{0} \in(0,1]$. If $t_{0} \in(0,1)$ then $y_{n}^{\prime \prime}\left(t_{0}\right)-\beta^{\prime \prime}\left(t_{0}\right) \leqq 0$. However since $y_{n}\left(t_{0}\right)>\beta\left(t_{0}\right)$ we have

$$
y_{n}^{\prime \prime}\left(t_{0}\right)-\beta^{\prime \prime}\left(t_{0}\right)=-\left[f\left(t_{0}, \beta\left(t_{0}\right)\right)+r\left(\beta\left(t_{0}\right)-y_{n}\left(t_{0}\right)\right)+\beta^{\prime \prime}\left(t_{0}\right)\right]>0,
$$

a contradiction. If $t_{0}=1$ then $y_{n}^{\prime}(1) \geqq \beta^{\prime}(1)$. However since $y_{n}(1)>\beta(1)$ we have

$$
y_{n}^{\prime}(1)-\beta^{\prime}(1)=\rho_{n}-\mu \psi^{* *}\left(y_{n}(1)\right)-\beta^{\prime}(1)=\rho_{n}-\left[\mu \psi(\beta(1))+\beta^{\prime}(1)\right]<\rho_{n}-\rho_{3} \leqq 0,
$$

a contradiction. Thus $(2.28)$ is true. Consequently

$$
\rho_{n} \leqq y_{n}(t) \leqq \beta(t) \text { for } t \in[0,1]
$$

Essentially the same reasoning as in Theorem 2.1 establishes

$$
\alpha(t) \leqq y_{n}(t) \leqq \beta(t) \text { for } t \in[0,1]
$$

In particular $\alpha(1) \leqq y_{n}(1) \leqq \beta(1)$ so $y_{n} \in C^{1}[0,1] \cap C^{2}(0,1)$ is a solution of $(2.25)^{n}$. The reasoning in Theorem 2.1 (from (2.18) onwards) now establishes that (2.1) has a solution.

We now discuss condition (2.6). One can usually "construct" $\alpha$ explicitly from the differential equation; see $[2,10,11]$. However if $(2.5)$ is replaced by the conditions 


$$
\left\{\begin{array}{l}
\text { let } n \in\{3,4, \ldots\} \text { and associated with each } n \text { we have a constant } \\
\rho_{n} \text { such that }\left\{\rho_{n}\right\} \text { is a decreasing sequence with } \lim _{n \rightarrow \infty} \rho_{n}=0, \\
\text { and there exists a constant } k_{0}>0 \text { such that for } \frac{1}{n} \leqq t \leqq 1-\frac{1}{n} \text { and } \\
0<y \leqq \rho_{n} \text { we have } f(t, y) \geqq k_{0}
\end{array}\right.
$$

and

$$
\psi(0)=0
$$

then we can construct an explicit $\alpha$ off the sequence of constants $\left\{\rho_{n}\right\}$; this is a standard argument, see [6] for example.

The details are as follows. Let $0 \leqq x \leqq \frac{1}{3}$ and

$$
r_{0}(x)=\left\{\begin{array}{l}
\rho_{k}\left(x-\frac{1}{k}\right)+\sum_{m=k+1}^{\infty} \rho_{m}\left(\frac{1}{m-1}-\frac{1}{m}\right), \quad x \in\left(\frac{1}{k}, \frac{1}{k-1}\right], \quad k=4,5, \ldots \\
0, \quad x=0
\end{array}\right.
$$

Remark. Notice $r_{0}(x)=\int_{0}^{x} \phi(s) d s$ for $0 \leqq x \leqq \frac{1}{3}$ where $\phi:\left[0, \frac{1}{3}\right] \rightarrow[0, \infty)$ is the step function defined by

$$
\phi(t)=\left\{\begin{array}{l}
0, \quad t=0 \\
\rho_{k}, k \in\left(\frac{1}{k}, \frac{1}{k-1}\right], \quad k=4,5
\end{array}\right.
$$

Here $r_{0} \in C\left[0, \frac{1}{3}\right]$ and notice

$$
r_{0}(t) \leqq \phi(t) \leqq \rho_{k} \quad \text { for } \quad t \in\left(\frac{1}{k}, \frac{1}{k-1}\right], \quad k=4,5, \ldots
$$

Next define

$$
\theta(t)=\int_{0}^{t} \int_{0}^{s} r_{0}(x) d x d s \quad \text { for } \quad 0 \leqq t \leqq \frac{1}{3}
$$

Notice $\theta(t) \leqq \rho_{k}$ for $t \in\left(\frac{1}{k}, \frac{1}{k-1}\right], k=4,5, \ldots$ and so

$$
f(t, y) \geqq k_{0} \quad \text { for } \quad(t, y) \in\left(0, \frac{1}{3}\right] \times\{y \in(0, \infty): y \leqq \theta(t)\}
$$

Let 


$$
\alpha^{*}(t)=\left\{\begin{array}{l}
\theta(t), 0 \leqq t<\frac{1}{3} \\
q(t), \frac{1}{3} \leqq t \leqq \frac{2}{3} \\
\theta(1-t), \frac{2}{3}<t \leqq 1
\end{array}\right.
$$

Here $q:\left[\frac{1}{3}, \frac{2}{3}\right] \rightarrow\left(0, \rho_{3}\right]$ is such that $q \in C^{2}\left[\frac{1}{3}, \frac{2}{3}\right]$ with $q\left(\frac{1}{3}\right)=\theta\left(\frac{1}{3}\right)=q\left(\frac{2}{3}\right), q^{\prime}\left(\frac{1}{3}\right)=\theta^{\prime}\left(\frac{1}{3}\right)=$ $-q^{\prime}\left(\frac{2}{3}\right)$ and $q^{\prime \prime}\left(\frac{1}{3}\right)=\theta^{\prime \prime}\left(\frac{1}{3}\right)=q^{\prime \prime}\left(\frac{1}{3}\right)$.

Notice since $0<q(t) \leqq \rho_{3}$ for $t \in\left[\frac{1}{3}, \frac{2}{3}\right]$ we have

$$
f(t, y) \geqq k_{0} \quad \text { for } \quad(t, y) \in\left[\frac{1}{3}, \frac{2}{3}\right] \times\{y \in(0, \infty): y \leqq q(t)\}
$$

Consequently (2.33) and (2.34) imply

$$
f(t, y) \geqq k_{0} \quad \text { for } \quad(t, y) \in(0,1) \times\left\{y \in(0, \infty): y \leqq \alpha^{*}(t)\right\}
$$

Finally define

$$
\alpha(t)=\eta \alpha^{*}(t)
$$

where

$$
\eta=\min \left\{1, \frac{k_{0}}{\left|\left(\alpha^{*}\right)^{\prime \prime}\right|_{0}+1}\right\}
$$

Now $\alpha \in C^{2}[0,1]$ with $\alpha(0)=0$ and $\alpha>0$ on $(0,1)$. Also since $\alpha(t) \leqq \alpha^{*}(t)$ we have

$$
f(t, y) \geqq k_{0} \quad \text { for } \quad(t, y) \in(0,1) \times\{y \in(0, \infty): y<\alpha(t)\} .
$$

In addition for $(t, y) \in(0,1) \times\{y \in(0, \infty): y<\alpha(t)\}$ we have

$$
f(t, y)+\alpha^{\prime \prime}(t) \geqq k_{0}+\alpha^{\prime \prime}(t) \geqq k_{0}-\frac{k_{0}\left|\left(\alpha^{*}\right)^{\prime \prime}(t)\right|}{\left|\left(\alpha^{*}\right)^{\prime \prime}\right|_{0}+1}>k_{0}-k_{0}=0 .
$$

Finally since $\left(\alpha^{*}\right)^{\prime}(t)=-\int_{0}^{1-t} r_{0}(x) d x$ for $\frac{2}{3} \leqq t \leqq 1$ we have $\left(\alpha^{*}\right)^{\prime}(1)=0$ and so $\alpha^{\prime}(1)+$ $\mu \psi(\alpha(1))=0+\mu \psi(0)=0$ since $(2.32)$ holds.

Consequently we have constructed an $\alpha$ which satisfies (2.6). However since (2.7) must also be satisfied it is desirable to construct the "best" $\alpha$. Usually it is possible to obtain an explicit $\alpha$ from the differential equation. We now provide a general result for the boundary value problem

$$
\left\{\begin{array}{l}
y^{\prime \prime}+f(t, y)=0,0<t<1 \\
y(0)=0 \\
y^{\prime}(1)-\mu y(1)=0,0 \leqq \mu<1
\end{array}\right.
$$

Remark. Note (2.37) is a special case of $(2.1)$; here $\psi(u)=-u$. 
Theorem 2.3. Suppose (2.2), (2.4), (2.8) and (2.31) are satisfied. In addition assume

$$
\left\{\begin{array}{l}
\text { there exists a function } \beta \in C[0,1] \cap C^{1}(0,1] \cap C^{2}(0,1) \text { with } \beta(0) \geqq \rho_{3}, \\
\beta^{\prime}(1)-\mu \beta(1)>\rho_{3}, \beta \geqq \rho_{3} \text { on }(0,1) \text { and } \beta(1)>\rho_{3} \\
\text { such that } f(t, \beta(t))+\beta^{\prime \prime}(t) \leqq 0 \text { for } t \in(0,1)
\end{array}\right.
$$

and

$$
\text { there exists } \tau \in(0,1) \text { with } f(t, y)>0 \text { for } t \in[\tau, 1) \text { and } 0<y \leqq \frac{\rho_{3}}{1-\mu(1-\tau)}
$$

are satisfied. Then (2.37) has a solution in $C[0,1] \cap C^{1}(0,1] \cap C^{2}(0,1)$.

Proof. Let $\gamma$ denote the $\alpha$ given above (i.e. as in (2.36)). Without loss of generality assume $\frac{1}{3}<\tau \leqq \frac{2}{3}$. Define

$$
\mu_{1}(t)=\left\{\begin{array}{l}
\gamma(t), 0 \leqq t \leqq \frac{1}{3} \\
\omega(t), \frac{1}{3}<t<\tau \\
\frac{\gamma(\tau)[1-\mu(1-t)]}{1-\mu(1-\tau)}, t \geqq \tau
\end{array}\right.
$$

and

$$
\alpha(t)=\eta \mu_{1}(t)
$$

where

$$
\eta=\min \left\{1, \frac{k_{0}}{\left|\mu_{1}^{\prime \prime}\right|_{0}+1}\right\}
$$

Here $\omega:\left[\frac{1}{3}, \tau\right] \rightarrow\left(0, \rho_{3}\right]$ is such that $\omega \in C^{2}\left[\frac{1}{3}, \tau\right]$ with

$$
\omega\left(\frac{1}{3}\right)=\gamma\left(\frac{1}{3}\right), \omega^{\prime}\left(\frac{1}{3}\right)=\gamma^{\prime}\left(\frac{1}{3}\right), \omega^{\prime \prime}\left(\frac{1}{3}\right)=\gamma^{\prime \prime}\left(\frac{1}{3}\right), \omega(\tau)=\gamma(\tau), \omega^{\prime}(\tau)=\frac{\mu \gamma(\tau)}{1-\mu(1-\tau)} \text { and } \omega^{\prime \prime}(\tau)=0
$$

Remark. Notice

$$
\alpha(1) \leqq \frac{\gamma(\tau)}{1-\mu(1-\tau)} \leqq \frac{\rho_{3}}{1-\mu(1-\tau)}
$$

We now claim that $\alpha$ satisfies (2.6). First notice $\alpha(0)=\alpha^{\prime}(1)-\mu \alpha(1)=0$. Also $\alpha>0$ on $(0,1)$ since $0 \leqq \mu<1$. Also from above

$$
f(t, y)+\alpha^{\prime \prime}(t)>0 \text { for }(t, y) \in(0, \tau) \times\{y \in(0, \infty): y<\alpha(t)\} .
$$


Now for $t>\tau$ we have $\alpha^{\prime \prime}(t)=0$ and also

$$
\alpha(t) \leqq \frac{\gamma(\tau)}{1-\mu(1-\tau)} \leqq \frac{\rho_{3}}{1-\mu(1-\tau)}
$$

Consequently

$$
f(t, y)+\alpha^{\prime \prime}(t)=f(t, y)>0 \quad \text { for } \quad(t, y) \in[\tau, 1) \times\{y \in(0, \infty): y<\alpha(t)\} .
$$

Thus (2.41) and (2.42) imply that $\alpha$ satisfies (2.6). Notice also that (2.7) is trivially satisfied since $\mu_{1}(t) \geqq \gamma(\tau)$ for $t>\tau$. Existence of a solution is now guaranteed from Theorem 2.2.

Example 2.1. (Membrane response of a spherical cap).

The boundary value problem

$$
\left\{\begin{array}{l}
y^{\prime \prime}+\left(\frac{t^{2}}{32 y^{2}}-\frac{\kappa^{2}}{8}\right)=0,0<t<1 \\
y(0)=0 \\
y^{\prime}(1)-\mu y(1)=0,0 \leqq \mu<1, \kappa>0
\end{array}\right.
$$

has a solution.

We will apply Theorem 2.3. First choose $n_{0} \in\{3,4, \ldots\}$ such that

$$
\frac{1}{2 n_{0}\left(8+\kappa^{2}\right)}<\frac{1-\mu}{2(1+\mu) \kappa}
$$

Notice that (2.31), with

$$
\rho_{n}=\frac{1}{2\left(n_{0}+n\right)\left(\kappa^{2}+8\right)^{1 / 2}} \text { and } k_{0}=1
$$

is true since for $\frac{1}{n} \leqq t \leqq 1-\frac{1}{n}$ and $0<y \leqq \rho_{n}$ we have

$$
f(t, y) \geqq \frac{1}{32 n^{2} \rho_{n}^{2}}-\frac{\kappa^{2}}{8} \geqq \frac{\left(\kappa^{2}+8\right)}{8}-\frac{\kappa^{2}}{8}=1 .
$$

Now let

$$
\beta(t)=\frac{t}{2 \kappa}+\rho_{3}
$$

Notice $\beta \geqq \rho_{3}$ on $[0,1]$ and $\beta^{\prime}(1)-\mu \beta(1)>\rho_{3}$ 


$$
\left(\text { i.e. } \frac{1}{2 \kappa}-\mu\left(\frac{1}{2 \kappa}+\rho_{3}\right)>\rho_{3}\right) \text { since } \rho_{3}<\frac{1-\mu}{2(1+\mu) \kappa} \text {. }
$$

In addition $f(t, \beta(t))+\beta^{\prime \prime}(t)=f(t, \beta(t)) \leqq 0$ on $(0,1)$ and so $(2.38)$ holds. Also let

$$
q_{1}(t)=\frac{t^{2}}{32}, g(y)=y^{-2}, q_{2}(t)=\frac{\kappa^{2}}{8} \text { and } h(y)=1
$$

so (2.4) and (2.8) are clearly true. Let

$$
\tau=\frac{1}{\left(n_{0}+3\right)(1-\mu)} \text { so for } \tau \leqq t<1 \text { and } 0<y \leqq \frac{\rho_{3}}{1-\mu(1-\tau)}
$$

we have

$$
f(t, y) \geqq \frac{\tau^{2}(1-\mu(1-\tau))^{2}}{32 \rho_{3}^{2}}-\frac{\kappa^{2}}{8} \geqq \frac{4 \tau^{2}(1-\mu)^{2}\left(n_{0}+3\right)^{2}\left(8+\kappa^{2}\right)}{32}-\frac{\kappa^{2}}{8}=1>0
$$

and so (2.39) is satisfied. Existence of a solution to (2.43) is now guaranteed from Theorem 2.3.

Our next two results are modelled on the Stefan boundary condition $y^{\prime}(1)+c y^{4}(1)=$ $0, c>0$.

Theorem 2.4. Suppose (2.2), (2.4), (2.5), (2.6), (2.7) and (2.8) hold. In addition suppose the following conditions are satisfied:

$\psi: \mathbf{R} \rightarrow \mathbf{R}$ is continuous and nondecreasing with $\psi(0)=0$ and $\psi(u)>0$ for $u>0$ and

$$
\left\{\begin{array}{l}
\text { there exists a constant } M>0 \text { such that for } z>0 \\
\int_{0}^{z} \frac{d u}{g(u)} \leqq \int_{0}^{1} x q_{1}(x) d x+\frac{h(z)}{g(z)} \int_{0}^{1} x q_{2}(x) d x+\int_{0}^{\rho_{3}} \frac{d u}{g(u)} \\
\text { implies } z \leqq M
\end{array}\right.
$$

Then (2.1), with $\mu>0$, has a solution in $C[0,1] \cap C^{1}(0,1] \cap C^{2}(0,1)$.

Remark. The case $\mu=0$, is included in Theorems 2.1 and 2.2.

Proof. Fix $n \in\{3,4, \ldots\}$ and look at 


$$
\left\{\begin{array}{l}
y^{\prime \prime}+f(t, y)=0,0<t<1 \\
y(0)=\rho_{n} \\
y^{\prime}(1)+\mu \psi(y(1))=\mu \psi\left(\rho_{n}\right) .
\end{array}\right.
$$

To show $(2.46)^{n}$ has a solution we consider the family of problems

$$
\left\{\begin{array}{l}
y^{\prime \prime}+\lambda f^{*}(t, y)=0,0<t<1 \\
y(0)=\rho_{n} \\
y^{\prime}(1)+y(1)+\lambda \psi_{n}^{*}(y(1))=\rho_{n}
\end{array}\right.
$$

where $f^{*}$ is as in Theorem 2.1 and

$$
\psi_{n}^{*}(z)=\left\{\begin{array}{l}
\mu\left[\psi(z)-\psi\left(\rho_{n}\right)\right]+\rho_{n}-z, z \geqq \rho_{n} \\
0, \quad z<\rho_{n} .
\end{array}\right.
$$

We first show

$$
y(t) \geqq \rho_{n}, \quad t \in[0,1]
$$

for any solution $y \in C^{1}[0,1] \cap C^{2}(0,1)$ to $(2.47)_{\lambda}^{n}$. To see this suppose $y-\rho_{n}$ has a negative minimum at $t_{0} \in(0,1]$. If $t_{0} \in(0,1)$ then we obtain a contradiction as in Theorem 2.1. It remains to consider the case $t_{0}=1$. Then $y^{\prime}(1) \leqq 0$. However since $y(1)<\rho_{n}$ we have

$$
y^{\prime}(1)=\rho_{n}-y(1)-\lambda \psi_{n}^{*}(y(1))=\rho_{n}-y(1)>0,
$$

a contradiction. Thus (2.48) holds.

Next suppose the absolute minimum of $y$ occurs at $t_{n} \in[0,1]$. In fact we may take $t_{n} \in(0,1)$, and so $y^{\prime}\left(t_{n}\right)=0$. To see this notice if $y\left(t_{n}\right)=\rho_{n}$ then $y \equiv \rho_{n}$. Next if $y\left(t_{n}\right)>\rho_{n}$ then if $t_{n}=1$ we have $y^{\prime}(1) \geqq 0$ and so

$$
y^{\prime}(1)=\rho_{n}-y(1)-\lambda \psi_{n}^{*}(y(1))=(1-\lambda)\left(\rho_{n}-y(1)\right)+\lambda \mu\left[\psi\left(\rho_{n}\right)-\psi(y(1))\right]<0,
$$

a contradiction.

For $x \in(0,1)$ we have

$$
\frac{-y^{\prime \prime}(x)}{g(y(x))} \leqq q_{1}(x)+q_{2}(x) \frac{h(y(x))}{g(y(x))}
$$

Integrate from $t\left(t<t_{n}\right)$ to $t_{n}$ and then from 0 to $t_{n}$ to obtain

$$
\int_{0}^{y\left(t_{n}\right)} \frac{d u}{g(u)} \leqq \int_{0}^{1} x q_{1}(x) d x+\frac{h\left(y\left(t_{n}\right)\right)}{g\left(y\left(t_{n}\right)\right)} \int_{0}^{1} x q_{2}(x) d x+\int_{0}^{\rho_{3}} \frac{d u}{g(u)}
$$


Consequently (2.45) implies

$$
\rho_{n} \leqq y(t) \leqq M \quad \text { for } \quad t \in[0,1]
$$

Define the mappings

$$
L, F: C_{\rho_{n}}^{1}[0,1] \rightarrow C_{0}[0,1] \times \mathbf{R}
$$

by

$$
L y(t)=\left(y^{\prime}(t)-y^{\prime}(0), \rho_{n}-y^{\prime}(1)-y(1)\right) \quad \text { and } \quad F y(t)=\left(-\int_{0}^{t} f^{*}(x, y(x)) d x, \psi_{n}^{*}(y(1))\right) .
$$

If $L y=(u(t), \gamma)$ then

$$
y(t)=\rho_{n}-\frac{t}{2}\left(\gamma+u(1)+\int_{0}^{1} u(x) d x\right)+\int_{0}^{t} u(x) d x
$$

hence $L^{-1}$ exists and is continuous. Also $F$ is completely continuous. Essentially the same reasoning as in Theorem 2.1 implies $(2.47)_{1}^{n}$ has a solution $y_{n} \in C^{1}[0,1] \cap C^{2}(0,1)$. Also $\rho_{n} \leqq y_{n}(t) \leqq M$ for $t \in[0,1]$, so $y_{n}$ is a solution of $(2.46)^{n}$.

Next we show

$$
\alpha(t) \leqq y_{n}(t) \leqq M \quad \text { for } \quad t \in[0,1]
$$

If this is not true then $y_{n}-\alpha$ would have a negative minimum at say $t_{0} \in(0,1]$. If $t_{0} \in(0,1)$ then we obtain a contradiction as in Theorem 2.1. If $t_{0}=1$ then $y_{n}^{\prime}(1) \leqq \alpha^{\prime}(1)$ and $0<y_{n}(1)<\alpha(1)$. However

$$
y_{n}^{\prime}(1)-\alpha^{\prime}(1)=\mu \psi\left(\rho_{n}\right)-\mu \psi\left(y_{n}(1)\right)+\mu \psi(\alpha(1)) \geqq \mu \psi\left(\rho_{n}\right)>0,
$$

a contradiction. Hence (2.50) is true.

Essentially the same reasoning as in Theorem 2.1 (from (2.18) onwards) now establishes the result.

Theorem 2.5. Suppose (2.2), (2.4), (2.5), (2.6), (2.7), (2.8) and (2.44) hold. In addition assume

$$
\left\{\begin{array}{l}
\text { there exists a function } \beta \in C[0,1] \cap C^{1}(0,1] \cap C^{2}(0,1) \text { with } \\
\beta \geqq \rho_{3} \text { on }[0,1], \beta^{\prime}(1)+\mu \psi(\beta(1)) \geqq \mu \psi\left(\rho_{3}\right) \text { and such that } \\
f(t, \beta(t))+\beta^{\prime \prime}(t) \leqq 0 \text { for } t \in(0,1)
\end{array}\right.
$$

Then (2.1), with $\mu>0$, has a solution in $C[0,1] \cap C^{1}(0,1] \cap C^{2}(0,1)$. 
Proof. Fix $n \in\{3,4, \ldots\}$ and look at $(2.46)^{n}$. The idea is to first consider

$$
\left\{\begin{array}{l}
y^{\prime \prime}+f^{* *}(t, y)=0,0<t<1 \\
y(0)=\rho_{3} \\
y^{\prime}(1)+y(1)+\psi_{n}^{* *}(y(1))=\rho_{n}
\end{array}\right.
$$

where $f^{* *}$ is as in Theorem 2.2 and

$$
\psi_{n}^{* *}(z)=\left\{\begin{array}{l}
\mu\left[\psi(\beta(1))-\psi\left(\rho_{n}\right)\right]+\rho_{n}-\beta(1), z>\beta(1) \\
\mu\left[\psi(z)-\psi\left(\rho_{n}\right)\right]+\rho_{n}-z, \rho_{n} \leqq z \leqq \beta(1) \\
0, \quad z<\rho_{n}
\end{array}\right.
$$

Let

$$
L, F: C_{\rho_{n}}^{1}[0,1] \rightarrow C_{0}[0,1] \times \mathbf{R}
$$

be defined by

$$
L y(t)=\left(y^{\prime}(t)-y^{\prime}(0), \rho_{n}-y^{\prime}(1)-y(1)\right) \quad \text { and } \quad F y(t)=\left(-\int_{0}^{t} f^{* *}(x, y(x)) d x, \psi_{n}^{* *}(y(1))\right)
$$

Now $F$ is compact so essentially the same reasoning as in Theorem 2.2 implies $(2.52)^{n}$ has a solution $y_{n} \in C^{1}[0,1] \cap C^{2}(0,1)$.

The reasoning in Theorem 2.4 yields

$$
y_{n}(t) \geqq \rho_{n} \text { for } \quad t \in[0,1] .
$$

Next we claim

$$
y_{n}(t) \leqq \beta(t) \text { for } t \in[0,1]
$$

If (2.54) is not true then $y_{n}-\beta$ would have a positive maximum at say $t_{0} \in(0,1]$. If $t_{0} \in(0,1)$ then we obtain a contradiction as in Theorem 2.2. If $t_{0}=1$ then $y_{n}^{\prime}(1) \geqq \beta^{\prime}(1)$ and $y_{n}(1)>\beta(1)$. However

$$
\begin{aligned}
y_{n}^{\prime}(1)-\beta^{\prime}(1) & \leqq\left[\rho_{n}-y_{n}(1)-\psi^{* *}\left(y_{n}(1)\right)\right]+\mu \psi(\beta(1))-\mu \psi\left(\rho_{3}\right) \\
& =\left(\beta(1)-y_{n}(1)-\mu\left[\psi(\beta(1))-\psi\left(\rho_{n}\right)\right]\right)+\mu \psi(\beta(1))-\mu \psi\left(\rho_{3}\right) \\
& =\beta(1)-y_{n}(1)+\mu\left[\psi\left(\rho_{n}\right)-\psi\left(\rho_{3}\right)\right]<0,
\end{aligned}
$$

a contradiction. Thus $(2.54)$ is true.

Hence $y_{n}$ is a solution of $(2.46)^{n}$. The same reasoning as in Theorem 2.4 establishes

$$
\alpha(t) \leqq y_{n}(t) \leqq \beta(t) \text { for } t \in[0,1]
$$


Essentially the same argument as in Theorem 2.1 (from (2.18) onwards) now establishes the result.

\section{REFERENCES}

1. J. V. BAXLEY, A singular nonlinear boundary value problem: membrane response of a spherical cap, SIAM J. Appl. Math. 48 (1988), 497-505.

2. L. E. Bobisud, J. E. Calvert and W. D. Royalty, Some existence results for singular boundary value problems, Differential Integral Equations 6 (1993), 553-571.

3. L. E. Bobisud, D. O'Regan and W. D. Royalty, Solvability of some nonlinear boundary value problems, Nonlinear Anal. 12 (1988), 855-869.

4. J. A. Gatica, V. Oliker and P. Waltman, Singular nonlinear boundary value problems for second order ordinary differential equations, J. Differential Equations 79 (1989), 62-78.

5. A. Granas, R. B. Guenther and J. W. Lee, Some general existence principles in the Carathéodory theory of nonlinear differential systems. J. Math. Pures Appl. 70 (1991), 153-196.

6. P. Habets and F. Zanolin, Upper and lower solutions for a generalized Emden-Fowler equation, J. Math. Anal. Appl. 181 (1994), 684-700.

7. C. D. Luning and W. L. Perry, Iterative solutions of negative exponent Emden-Fowler problems, Internat. J. Math. Sci. 13 (1990), 159-164.

8. J. W. Mooney, Solution of a Thomas-Fermi problem using linear approximants, Comput. Phys. Comm. 96 (1993), 51-57.

9. T. Y. N $\mathrm{N}_{\mathrm{A}}$ Computational methods in engineering boundary value problems (Academic Press, New York, 1979).

10. D. O'Regan, Positive solutions to singular boundary value problems with at most linear growth, Appl. Anal. 49 (1993), 171-196.

11. D. O'Regan, Theory of singular boundary value problems (World Scientific, Singapore, 1994).

12. S. Taliaferro, A nonlinear singular boundary value problem, J. Nonlinear Anal. 3 (19790), 897-904.

Department of Mathematics

University College

GALWAY

IRELAND 